Speakers bureau: Prof. Grassi reports personal fees from AbbVie, personal fees from Celgene, personal fees from Grünenthal, personal fees from Pfizer, personal fees from Union Chimique Belge Pharma, outside the submitted work., Emilio Filippucci Speakers bureau: Dr. Filippucci reports personal fees from AbbVie, personal fees from Bristol-Myers Squibb, personal fees from Celgene, personal fees from Roche, personal fees from Union Chimique Belge Pharma, personal fees from Pfizer, outside the submitted work.

DOI: 10.1136/annrheumdis-2020-eular.1113

\section{SAT0032 INCIDENCE OF RHEUMATOID ARTHRITIS IN PATIENTS WITH NEW ONSET OF MUSCULOSKELETAL SYMPTOMS AND ANTI-CPP POSITIVITY COMPARED TO ANTI-CPP NEGATIVE PATIENTS}

S. Dauth ${ }^{1,2}$, M. Köhm ${ }^{1,2,3}$, T. Oberwahrenbrock ${ }^{1,2}$, U. Henkemeier ${ }^{1,2,4}$, T. Rossmanith ${ }^{1,2}$, K. Mergenthal ${ }^{5}$, J. J. Petersen ${ }^{5}$, H. Burkhardt ${ }^{1,2,3,4}$, F. Behrens ${ }^{1,2,3,4} .{ }^{1}$ Fraunhofer IME Translational Medicine and Pharmacology, Frankfurt, Germany; ${ }^{2}$ Fraunhofer Cluster of Excellence Immune-Mediated Diseases CIMD, Frankfurt, Germany; ${ }^{3}$ Goethe-University Frankfurt, Rheumatology Department, Frankfurt, Germany; ${ }^{4}$ Centre of Innovative Diagnostics and Therapeutics Rheumatology/Immunology CIRI, Frankfurt, Germany; ${ }^{5}$ Goethe-Unversity Frankfurt, Institute for General Practice, Frankfurt, Germany

Background: Rheumatoid Arthritis (RA) is a chronic inflammatory joint disease. Strategies for its early detection and diagnosis are of high importance as prompt treatment improves clinical and structural outcome. Autoantibodies against cyclic citrullinated proteins (anti-CCP) have been associated with RA-development. Non-specific musculoskeletal (nsMSK) symptoms are often described prior to RA development. Majority of patients with nsMSK symptoms present to their general practice (GP) first. Studies of early arthritis cohorts have shown that many early arthritis patients cannot be accurately diagnosed at their first visit and are often referred as undifferentiated arthritis patients.

Objectives: To evaluate the incidence of anti-CCP positivity in patients with new onset of nsMSK symptoms and the incidence of RA in these patients over a 3-year follow-up period compared to anti-CPP negative patients.

Methods: In this prospective study (PANORA), 978 patients with new onset of nsMSK symptoms were included in 77 GP sites in Germany. Patients with a positive anti-CCP rapid-test (CCPoint $\AA$ ) were referred to Rheumatology Department (RD) for rheumatological assessment, RA-evaluation and an anti-CCP validation test (ELISA). ELISA anti-CCP positive patients without RA were monitored every 6 months for a total follow-up of 36 months or until RA-diagnosis. Patients with a negative anti-CPP result (CCPoint $₫$ or ELISA) are followed up with a questionnaire after 1 and $3 \mathrm{y}$.

Results: From 978 included patients, 105 (10.7\%) were CCPoint $₫$ positive. 96 were tested with ELISA and 27 (28.1\%) were confirmed anti-CCP positive. 9 (33.3\%) were diagnosed with RA at the first RD visit (study visit 2); 4 further patients were diagnosed with RA during the follow-up (FU) period so far. Overall, 48.1\% of ELISA-positive (ELISA+) patients were diagnosed with RA up to now; $11 \mathrm{ELISA}+$ patients are still in the FU period of the study. Of the $868 \mathrm{CCPoint} \circledast$ negative patients, currently, 282 have filled out a 1-year FU questionnaire; $3.5 \%$ of those reported a RA diagnosis (Table 1). As expected, clinical parameters at V2 (e.g. CRP, swollen and tender joint count) were worse in the ELISA+/RA+ group compared to the ELISA-/RA- group, but no obvious differences were detected between ELISA+ patients who were diagnosed with RA during the FU period (after V2) and ELISA-/RA- patients

Table 1. Number and percentage of patients with a RA diagnosis

\begin{tabular}{|c|c|c|c|}
\hline Anti-CCP status & Visit 2 & Follow-up* & Total \\
\hline $\begin{array}{l}\text { Point-of-Care Test - } \\
\text { Point-of-Care Test + / ELISA - } \\
\text { Point-of-Care Test + / ELISA + }\end{array}$ & $\begin{array}{c}- \\
2.9 \%(2 \text { of } 69) \\
33.3 \%(9 \text { of } 27)\end{array}$ & $\begin{array}{c}3.5 \%(10 \text { of } 282)^{\#} \\
0 \%(0 \text { of } 34)^{\#} \\
14.8 \%(4 \text { of } 27)\end{array}$ & $\begin{array}{c}3.5 \%(10 \text { of } 282)^{\#} \\
2.9 \%(2 \text { of } 69) \\
48.1 \%(13 \text { of } 27)^{\$}\end{array}$ \\
\hline
\end{tabular}

* 1 year-questionnaire for Point-of-Care Test and ELISA negative patients or every 6 months follow-up for ELISA positive patients; ${ }^{\text {PPatient-reported; }}{ }^{\$} 11$ patients are still in the follow-up phase of the study

Conclusion: Currently, $48.1 \%$ of anti-CCP+ (ELISA) patients have received a RA diagnosis, whereas $3.5 \%$ of the anti-CCP- (CCPoint $\Theta)$ received a RA diagnosis (patient reported), which underlines, that anti-CCP can be used as a marker to identify high-risk patients in GP setting. While clinical parameters are correlated with the diagnosis of RA, they are not suited for predicting future RA development alone. Anti-CCP, possibly in combination with additional parameters imaging, might increase the likelihood to early diagnose or predict RA development.

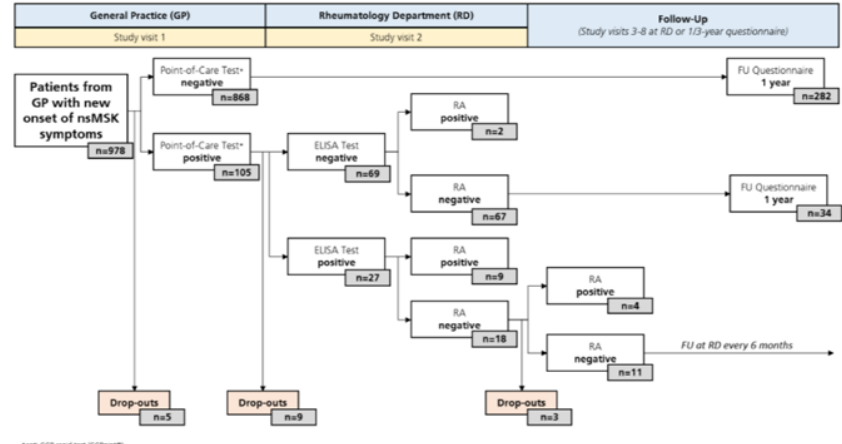

Figure 1. Study overview: Patient distribution depending on anti-CCP results and RA diagnosis.

Disclosure of Interests: Stephanie Dauth Grant/research support from: BMS Michaela Köhm Grant/research support from: Pfizer, Janssen, BMS, LEO, Consultant of: BMS, Pfizer, Speakers bureau: Pfizer, BMS, Janssen, Novartis Timm Oberwahrenbrock Grant/research support from: BMS, Ulf Henkemeier: None declared, Tanja Rossmanith Grant/research support from: Janssen BMS, LEO, Pfizer, Karola Mergenthal Grant/research support from: BMS, Juliana J. Petersen Grant/research support from: BMS, Harald Burkhardt Grant/ research support from: Pfizer, Roche, Abbvie, Consultant of: Sanofi, Pfizer, Roche, Abbvie, Boehringer Ingelheim, UCB, Eli Lilly, Chugai, Bristol Myer Scripps, Janssen, and Novartis, Speakers bureau: Sanofi, Pfizer, Roche Abbvie, Boehringer Ingelheim, UCB, Eli Lilly, Chugai, Bristol Myer Scripps, Janssen, and Novartis, Frank Behrens Grant/research support from: Pfizer, Janssen, Chugai, Celgene, Lilly and Roche, Consultant of: Pfizer, AbbVie, Sanofi, Lilly, Novartis, Genzyme, Boehringer, Janssen, MSD, Celgene, Roche and Chugai

DOI: 10.1136/annrheumdis-2020-eular.5463

\section{SAT0033 USING CHROMOSOME CONFORMATION FOR INSIGHT INTO PATHOGENESIS OF EARLY RHEUMATOID ARTHRITIS ENDOTYPES}

C. Duncan ${ }^{1}$, E. Hunter ${ }^{2}$, C. Koutsothanasi ${ }^{2}$, M. Salter ${ }^{2}$, A. Akoulitchev², I. Mcinnes', C. Goodyear' ${ }^{1}{ }^{1}$ University of Glasgow, Glasgow, United Kingdom; ${ }^{2}$ Oxford Biodynamics, Oxford, United Kingdom

Background: Rheumatoid arthritis (RA) is a chronic autoimmune disease with substantial immunopathogenic heterogeneity. It is well established that early diagnosis and initiation of effective therapy is crucial to prevent loss of function. Previously, various RA treatment trajectories have been identified, however there are currently no clinically validated biomarkers that can identify these trajectories at the start of treatment. Evaluation of the structural epigenome has revealed that chromosome conformation signatures (CCS) offer great potential as binary, informative biomarkers, and have been previously shown to predict early RA patient response to Methotrexate with $90 \%$ sensitivity (1). These signatures can also reveal highly regulated areas of the genome, which may be underpinning disease endotypes.

Objectives: The objective of this study was to evaluate the structural epigenome in early RA over longitudinal samples to determine whether it is associated with treatment trajectories.

Methods: Patient data and samples were from the Scottish Early Rheumatoid Arthritis (SERA) cohort; a pan-Scotland inception cohort. CDAI, DAS28 ESR and DAS28 CRP measurements were calculated at baseline, 6 months and 12 months to determine longitudinal treatment response. From 3 principal longitudinal response trajectories, 18 patients (who had equivalent disease activity at baseline) were chosen to investigate the structural epigenome. These 18 comprised of responders (R), non-responders (NR) and initial responders (IR; low disease activity/remission at 6 months but moderate/high disease activity at 12 months) with 6 patients per group at each time point. 20 pooled healthy samples were used as a comparator population. EpiSwitch libraries were probed on $180 \mathrm{~K}$ Agilent SureSelect custom arrays that were designed using EpiSwitch propriety information and publicly available data from Walsh et al (2). Microarray data was analysed using the Limma package within R studio.

Results: EpiSwitch array analysis showed that there were $>10,000$ statistically significant differential chromosomal loops between R, NR and IR. Evaluation of the 3 trajectory groups (R, NR and IR), taking into account the healthy chromosomal conformation, revealed an RA-associated structural epigenome that comprised of 10,445 chromosomal loops that were stable, over the three time points. Subsequent analysis of the distinct treatment 